\title{
Determination of the ball mills performance, taking into account the particle size of the finished product
}

\author{
Rashid Sharapov* \\ Moscow State University of Civil Engineering (National Research University), 129337, Moscow, \\ Russia
}

\begin{abstract}
The article discusses the issue of determining the newly formed specific surface of the cement clinker taking into account the lateral surface of the seal area. When developing this technique, the assumption that the newly formed particles have a shape close to spherical, has been made. The obtained ratio determines the work that considers the formation of thecement clinker material new surface, depending on the size of the original and newly formed material. An arithmetic expression is given, defined as the minimum amount of energy that must be introduced into the deformation zone for the destruction of a cement clinker particle to occur. The presented technique is specified taking into account the fact that defects are introduced into the crushed material particle. Taking into account the fact that the grinding ball makes an impact in one cycle of its movement, the value of the newly formed surface of the material in the ball mill is determined in one hour of its operation.
\end{abstract}

\section{Introduction}

Portland cement is one of the main building materials used in construction. At the same time, the production of Portland cement is characterized by both thermal and electrical high energy consumption $[1,2]$. For the production of cement, depending on the method of production, up to $150 \mathrm{~kW} \cdot \mathrm{h} / \mathrm{t}[1,3]$. One of the most energy-intensive processes in the production of cement is fine grinding of cement clinker and additives [1, 4, 5].

For fine grinding of cement clinker and additives, ball mills of various sizes are mostly used $[1,2,6,7]$. These grinding units are distinguished by the simplicity of their design, its operation and repair [8-12]. However, a significant amount of energy spent on fine grinding of clinker and additives forces a large number of researchers to offer to the market grinding units based on other grinding principles [13-16].

To improve the grinding process in ball mills, many authors propose various models of interaction of the ground medium with grinding bodies [17-21].

There is a work [22] in which the destruction of a clinker particle occurs due to the energy introduction into the particle by the grinding body. The work [23] considers the

\footnotetext{
*Corresponding author: ptdm_zavkaf@mail.ru
} 
destruction of particles as a result of a generalized thermodynamic force, due to the stretching of the particle itself when an external force is applied to it.

Taking into account the approach proposed above, when a grinding medium acts on a particle, the work the value of which can be found if we use the formula (8), will be performed [22]. To calculate the coefficient of energy expansion $\alpha$, included in this equation, we calculate the change in the volume of the seal area in the infinitesimal process of energy introduction $Q$. Therefore, we have:

$$
d V=d\left(\pi r^{2} h\right)=2 \pi r h d r+\pi r^{2} d h=2 \pi r^{2} h \frac{d r}{r}+\pi r^{2} h \frac{d h}{h}
$$

The relation (1) can be reduced to the form:

$$
d V=2 V_{0} d \varepsilon_{r}+V_{0} d \varepsilon_{h}
$$

It is taken into account here that $d \varepsilon_{r}=\frac{d r}{r}$, a $d \varepsilon_{h}=\frac{d h}{h}$.

Taking into account the expression (1), the relation (2) takes the form:

$$
d V=V_{0}\left(1-\frac{2}{\mu_{2}}\right) d \varepsilon_{h}
$$

Similarly, we find that

$$
d Q=-h d F
$$

The minus sign in the formula (4) means that with deepening into the seal area, the value of force $F$ is decreased. As

$$
d F=S(x) d \tau
$$

where $\tau$ is the compression stress, $\mathrm{Pa}$;

$$
d \tau=E_{2} d \varepsilon_{h} .
$$

Taking into account the equations (5) and (6), the expression (4) can be represented in the following form:

$$
d Q=-V_{0} E_{2} d \varepsilon_{h}
$$

Substitution of the equations (3) and (7) into the expression (3) [22] leads to the following value for the matter energy expansion coefficient in the seal area:

$$
\alpha=\frac{2-\mu_{2}}{\mu_{2} E_{2} V_{0}} .
$$


The expression for the generalized thermodynamic force fwill be found from the relation connecting the equality of the compressive and tensile stresses on the lateral surface of the seal area (the lateral surface of the cylinder with the radiusrand height $h$ ). Therefore, we have

$$
f=\sigma \frac{S_{d}}{S_{1}}
$$

Here $S_{1}$ is the lateral surface of the seal area, equal to:

$$
S_{1}=2 \pi r h=2 \pi \sqrt{\frac{2 \delta}{1+x}} \delta R_{2}^{2}=\pi \sqrt{\frac{8 \delta^{3}}{1+x}} R_{2}^{2}
$$

$S_{d}$ defines the total surface of cement clinker particles having an average diameter $d$, formed in the process of the original particle destruction, then:

$$
S_{d}=\pi d^{2} \cdot n_{1}
$$

Where $n_{1}$ is a number of newly formed particles'parts.

To determine the number of newly formed particles'parts, we proceed from the following assumption: the newly formed particles have a shape close to spherical (the assumption of a different shape, for example, cubic for both the original particle and the newly formed one, does not change the generality of reasoning for calculating the value $n_{1}$, but leads only to a different coefficient value).

$$
n_{1}=\frac{\frac{4}{3} \pi R_{2}^{3}}{\frac{\pi d^{3}}{6}}=\frac{8 R_{2}^{3}}{d^{3}} .
$$

Substitution of the equation (12) into the equation (11) leads to the following expression:

$$
S_{d}=\frac{8 R_{2}^{3}}{d}
$$

Taking into account the equations (13) and (10), the expression (9) can be represented in the following form:

$$
f=\sqrt{\frac{8(1+x)}{\delta^{3}}} \frac{R_{2}}{d} \sigma
$$

Applying the equations (14), 8), to the expression (8) [22] we find the value of the work done by the seal area during the expansion:

$$
A=\sqrt{\frac{8(1+x)}{\delta^{3}}} \cdot \frac{\left(2-\mu_{2}\right) \sigma}{\mu_{2} E_{2}} Q \frac{R_{2}}{d}-\frac{24 \pi\left(1-2 \mu_{2}\right) \sigma^{2}}{\delta d^{2} E_{2}} R_{2}^{5}
$$

The obtained relation (15) determines the work done as a result of the introduction into the deformation zone of the energy determined by the formula (15) [23], which is spent on 
the initial material destruction. On the other hand, the work that must be spent on the cement clinker destruction with the formation of a new surface $S_{0}$ can be written as follows:

$$
A=\gamma \cdot S_{0}
$$

where $\gamma$ is specific surface energy, $\mathrm{J} / \mathrm{m}^{2}$.

If we take into account that the work, given by the formula (15), is spent on the formation of a new surface $S_{0}$, then the obtained relations (15) and (16) make it possible to determine the analytical dependence of this surface depending on the technological parameters of the grinding plant. In view of the above-said, we obtain the expression that determines the formation of a new surface of the cement clinker material, depending on the original and newly formed material size.

$$
S_{0}=\frac{1}{\gamma}\left\{\sqrt{\frac{8(1+x)}{\delta^{3}}} \cdot \frac{\left(2-\mu_{2}\right) \tau}{\mu_{2} E_{2}} Q \frac{R_{2}}{d}-\frac{24 \pi\left(1-2 \mu_{2}\right) \sigma^{2}}{\delta d^{2} E_{2}} R_{2}^{5}\right\}
$$

After simple mathematical transformations, the relation (17) can be reduced to the form:

$$
S_{0}=\frac{2 \sigma R_{2}}{\gamma E_{2} d}\left\{\sqrt{\frac{2(1+x)}{\delta^{3}}} \cdot \frac{2-\mu_{2}}{\mu_{2}} Q-\frac{12 \pi\left(1-2 \mu_{2}\right)}{\delta} \frac{R_{2}^{4}}{d} \sigma\right\}
$$

From the relation (18), it is possible to obtain the condition for the cement clinker material destruction, which follows from the positiveness of the newly formed surface value:

$$
Q>\frac{12 \pi \mu_{2}\left(1-2 \mu_{2}\right) \delta^{\frac{1}{2}}}{\left(2-\mu_{2}\right) \sqrt{2(1+x)}} \cdot \frac{\sigma R_{2}^{4}}{d}
$$

The arithmetic expression on the right-hand side of inequality (19) can be interpreted as the minimum amount of energy that must be introduced into the deformation zone for the destruction of a cement clinker particle to occur. Therefore:

$$
Q_{\min }=\frac{12 \pi \mu_{2}\left(1-2 \mu_{2}\right) \delta^{\frac{1}{2}}}{\left(2-\mu_{2}\right) \sqrt{2(1+x)}} \frac{\sigma R_{2}^{4}}{d}
$$

The relation (20) determines the required minimum of energy that must be transferred to a particle of cement clinker for its destruction, depending on the characteristic parameters of grinding bodies, material, design and technological parameters of the mill.

Taking into account the equation (20), the relation (18) is represented in the form:

$$
S_{0}=\frac{2\left(2-\mu_{2}\right) \sqrt{2(1+x)}}{\delta^{\frac{3}{2}} \mu_{2}} \cdot \frac{\sigma R_{2} Q}{\gamma \cdot E_{2} d}\left(1-\frac{Q_{\text {min }}}{Q}\right)
$$

The expression (18) for the newly formed surface of a particle of crushed cement clinker can be expressed through the average size $d$ formed parts. For this, using the formula (12), we establish the required connection 


$$
R_{2}=\frac{\sqrt[3]{n}}{2} d
$$

and substitution of the equation (22) into the relation (18) taking into account the equations (11) and (12) [23] will lead to the following result:

$$
S_{0}=\frac{\sigma \sqrt[3]{n}}{2 \gamma \cdot E_{2}}\left\{\sqrt{\frac{8(1+y)}{\delta^{3}(y)}} \cdot \frac{2-\mu_{2}}{\mu_{2}} Q-\frac{3 \pi\left(1-2 \mu_{2}\right) n^{\frac{4}{3}} d^{3} \sigma}{2 \delta(y)}\right\}
$$

where

$$
\begin{gathered}
y=\frac{\sqrt[3]{n} \cdot d}{2 R_{1}} \\
\delta(y)=\left[\frac{5}{4} \pi A_{1} \cdot \rho_{2} v_{1}^{2} \frac{\sqrt{1+y}}{\left(1+\frac{\rho_{2}}{\rho_{1}} y^{3}\right)}\right]^{\frac{2}{5}} .
\end{gathered}
$$
form:

Taking into account the equations (24) and (25), the expression (23) is reduced to the

$$
S_{0}=\frac{\sqrt[3]{n} \cdot \sigma}{2 \gamma \cdot E_{2}} \sqrt{\frac{8(1+y)}{\delta^{3}(y)}} \cdot \frac{2-\mu_{2}}{\mu_{2}} Q\left[1-\left(\frac{d}{d_{m}(y)}\right)^{3}\right]
$$

Here

$$
d_{m}(y)=\left(\frac{2\left(2-\mu_{2}\right) \sqrt{8(1+y)}}{3 \pi \mu_{2}\left(1-2 \mu_{2}\right) \delta^{\frac{1}{2}}(y) n^{\frac{4}{3}}} \cdot \frac{Q}{\sigma}\right)^{\frac{1}{3}}
$$

The expression defined by the formula (27) can be interpreted as the largest particle size of the cement clinker, which is formed from the original particle of the material $R_{2}$ when hitting it with a grinding body of size $R_{1}$ when the latter decays into $n_{1}$ parts.

To determine the value of specific surface energy $\gamma$, included in the relation (16), we assume that a tensile stress acts on the cement clinker particle $\sigma$. If there are no defects (cracks) in the clinker, then the value of the stored deformation energy will be equal to:

$$
W_{0}=\frac{\sigma^{2} V}{E_{2}}
$$

where $V$ is a volume of the loading zone of the cement clinker particle.

In a real particle of cement clinker, there are always defects (cracks, boundaries of sections of the main clinker minerals and vitreous matter). If there is an elliptical crack in the cement clinker that $a$ and $b$, respectively, the major and minor semiaxes of the ellipse, then the concentration of elastic stresses occurs near it, and as a result of which there is an increase in the energy of elastic deformations by the value: 


$$
W_{1}=\frac{\chi \sigma^{2} a^{2} b}{E_{2}}
$$

here $\chi$ is the defect concentration factor in a clinker particle.

If we further assume that the size of the crack bin the direction perpendicular aremains constant, and the length begins to increase, then an increase in the energy value, according to the expression (29), will be spent on breaking intermolecular bonds, i.e., on the formation of a new surface:

$$
W_{2}=2 \gamma a b
$$

Therefore, on the basis of the energy conservation law, it is possible to write that:

$$
\frac{\sigma^{2} V}{E_{2}}+\frac{\chi \sigma^{2} a^{2} b}{E_{2}}=2 \gamma a b
$$

The condition for crack growth (the length of the crack, starting from which the destruction of the cement clinker particle occurs) is the equality to zero of the derivative with respect to $a$ from the relation (31):

$$
\frac{2 \chi \sigma^{2} a b}{E_{2}}=2 \gamma b
$$

From the relation (32) we determine the critical crack length:

$$
a_{\mathrm{\kappa}}=\frac{\gamma E_{2}}{\chi \sigma^{2}}
$$

From the expression (33) we find that:

$$
\gamma=\frac{\chi a \sigma^{2}}{E_{2}}
$$

The composition $\chi a$, having the length size, can be called the grinding parameter, the value of which for a cement clinker particle can be determined experimentally.

Taking into account the equation (34), the relation (18) can be represented in the following form:

$$
S_{0}=\frac{2 R_{2}}{\chi a \sigma d}\left\{\sqrt{\frac{2(1+x)}{\delta^{3}}} \cdot \frac{2-\mu_{2}}{\mu_{2}} Q-\frac{12 \pi\left(1-2 \mu_{2}\right)}{\delta} \cdot \frac{R_{2}^{4}}{d} \sigma\right\}
$$

Thus, the expression (13) determines the size of the newly formed surface in a single interaction of a metal ball with a radius $R_{1}$ with a particle of cement clinker when changing the latter from $R_{2}$ up to $d$.

At the next impact of the ball on a crushed particle with a diameter $d$, is assigned a value $R_{2}$ in the formula (15) and the following value $d$ is determined. 
Within one hour, the clinker particle will perceivekblows from the condition that the ball makes a blow in one cycle of its movement. This cycle is made up of time $t_{1}$ the movement of the ball along a circular path along with the drum of the mill and time $t_{2}$ the movement of balls along a parabolic trajectory in their free fall. Taking this into account, the number of impacts of the ball on the clinker particle within one hour is determined by the formula

$$
k=\frac{3600}{t_{1}+t_{2}}
$$

Taking into account the recommendations of M. Ya. Sapozhnikov [24], we determine the parameter $k$ for a ball mill from the ratio:

$$
t_{1}+t_{2}=\frac{60 \cdot 0.692}{n_{1}}
$$

where $n_{1}$ is the mill drum rotation speed, rot $/ \mathrm{min}$.

Then the relation (2.73) takes the form:

$$
k=\left[\frac{3600 \cdot n_{1}}{60 \cdot 0.692}\right] \cong 87 n_{1}
$$

To define the newly formed surface Sparticles of the original cement clinker, we find the number of cement clinker particles $N$ to be destroyed. From the practice of operating ball mills, it is known that the particles of the crushed material are in the voids between the grinding bodies. Obviously, based on the above-said, the parameter Nis determined from the relation:

$$
N=\frac{V_{l}-V_{b} n_{2}}{V_{p}}
$$

where $V_{l}$ is loading volume of a ball mill with a clear radius $R_{0}$ and length $L_{0}$, which is determined according to:

$$
V_{l}=\pi R^{2}{ }_{0} L_{0} \varphi
$$

Here $\varphi$ is a ball mill load factor; $V_{b}$ is sphere volume, $\mathrm{m}^{3} ; n_{2}$ is a number of grinding balls in the drum of the mill, pcs.; $V_{p}$ is volume of a clinker particle to be crushed, $\mathrm{m}^{3}$.

Substitution of the expression (39) into the equation (38), taking into account the sizes of grinding bodies and particles of ground materials, leads to the relation:

$$
N=\frac{\pi R_{0}^{2} L_{0} \phi-\frac{4}{3} \pi R_{1}^{3} n_{2}}{\frac{4}{3} \pi R_{2}^{3}}
$$

The relation (40) obtained above can be conveniently reduced to the following form:

$$
N=\frac{3}{4} \frac{\phi R_{0}^{2} L_{0}}{R_{2}^{3}}-\left(\frac{R_{1}}{R_{2}}\right)^{3} n_{2}
$$


Thus, as a result of the destruction $N$ particles of cement clinker for the number of cycles determined by the relation (37) at $n$-multiple grinding forms a finished product with a total newly formed surface:

$$
S_{N}=S_{0} \cdot N=\frac{3}{4} \frac{\varphi R_{0}^{2} L_{0}}{R_{2}^{3}}\left(\frac{R_{1}}{R_{2}}\right)^{3} n_{2} \cdot S_{0}
$$

The resulting relation (35) also makes it possible to find the value of the newly formed surface if as $Q$ put the value of the useful work, which is necessary to grind the material from the original size $R_{2}$ to the final $d$.

Therefore, if the equation (35) is substituted, then:

$$
Q=A_{n}=\eta_{l} \cdot A_{l}=\eta_{l} N_{l} \cdot t
$$

If in the relation (43) the value $t(t=3600 \mathrm{~s})$ is equal to one hour, then, taking into account the equation (43), we find the value of the newly formed surface of the material in the ball mill for one hour of its operation:

$$
S_{N}=\frac{2 R_{2} N}{\chi a_{k} \sigma d}\left\{\sqrt{\frac{2(1+x)}{\delta^{3}}} \cdot \frac{2-\mu_{2}}{\mu_{2}} \eta_{b} P \cdot 3600-\frac{12 \pi\left(1-2 \mu_{2}\right)}{\delta} \cdot \frac{R_{2}^{4}}{d} \sigma\right\}
$$

where $P$ defines the ball mill drive power; $\eta_{b}$ is a ball mill performance coefficient.

\section{Conclusions}

Thus, the obtained expression makes it possible to determine the productivity of the ball mill on the newly formed surface per hour of its operation.

\section{References}

1. W.H. Duda, Cement-data-book, Band 1, Internationale Verfahrenstechniken der Zementindustrie (Wiesbaden, Bauverlag, Berlin, 1985).

2. V.A. Perov, E.E. Andreev and L.F. Bilenko, Crushing, grinding and screening of minerals (Nedra, Moscow, 1990).

3. Y.M. Butt and V.V. Timashov, Chemical technology of binders (Higher school, Moscow, 1980).

4. A. Nerholm, Grinding of cement, Symposium on cement manufacture (Niicement Research Institute, Moscow, 1979).

5. R.R. Sharapov, Matrix model of the grinding process of cement clinker in the ball mill, International Conference on Mechanical Engineering and Applied Composite Materials (MEACM) Hong Kong, November 23-24, 2017. International conference on Mechanical Engineering and Applied Composite Materials, 307.

6. V.S. Sevostyanov, A.A. Goncharov and V.P. Potelezhko, Glass and Ceramics 50 (2), 55-59 (1993).

7. Global Projects World Cement December, 87-90 (2007|). 
8. V.S. Bogdanov, S.I. Hanin, D.N. Starchenko, I.A. Sagitov, Journal of Engineering and Applied Sciences 9 (11), 2344-2350 (2014).

9. V.S. Bogdanov, M.Y. Eltsov, L.Y. Shirokova, P.A. Khakhalev, ZKG International 70 (5), 64-66 (2017).

10. R.R. Sharapov, I.V. Shrubchenko and A.M. Agarkov, International Journal of Applied Engineering Research 10 (12), 31341-31348 (2015).

11. V.S. Sevost'yanov, I.I. Targonskij, A.A. Romanovich, A.A. Goncharov, Glass andCeramics 4, 28-30 (1993).

12. V.S. Sevost'yanov, A.A. Goncharov, V.P. Potelezhko, Glass and Ceramics 50 (2), 55 59 (1993).

13. H. Brundiek, Zement - Kalk - Gips. - International 53, 177-185 (2000).

14. O. Jung, Cement International 2, 52-57 (2004).

15. R. de la Foucyardiere, Cement International 56, 44-49 (2003).

16. Y. Reinchardt, Effective Finish Grinding. World Cement, March (2008) 93-95.

17. I.G.Luginina, I.N.Novoselova and A.G.Novosyolov, Middle East Journal of Scientific Research 18 (11), 1676-1683 (2013).

18. M.M. Kuznetsova, V.E. Ved, E.V. Krasnokutskiy, M.I. Satayev and A.A. Saipov, Chemical Engineering Transactions 61, 541-546 (2017).

19. A. Farzanegan, Ardi E. Ghasemi, V. Hasanzadeh and A.Valian, Journal of Central South University 21 (2), 799-810 (2014).

20. L.D.Shakhova, I.G.Luginina and R.A.Cherkasov, Modern Applied Science 8(6), 296305 (2014).

21. V.S. Bogdanov, S.I. Antsiferov, N.E. Bogdanov, Middle East Journal of Scientific Research 18 (10), 1448-1454 (2013).

22. R. R. Sharapov and E. V.Kharlamov, IOP Conf. Series: Materials Science and Engineering 698 (2019).

23. R.R. Sharapov, IOP Conference Series: Materials Science and Engineering 913 (4) (2020).

24. M.Ya. Sapohznikov, Mechanical equipment of enterprises of building materials, products and structures (Higher School, Moscow, 1971). 